\title{
EXTENDED LIE ALGEBRAIC STABILITY ANALYSIS FOR SWITCHED SYSTEMS WITH CONTINUOUS-TIME AND DISCRETE-TIME SUBSYSTEMS
}

\author{
Guisheng ZHAI* ${ }^{*}$ Xuping XU ${ }^{* *}$, Hai LIN ${ }^{* * *}$, Derong LIU ${ }^{* * * *}$ \\ * Department of Mechanical Engineering \\ Osaka Prefecture University \\ Sakai, Osaka 599-8531, Japan \\ e-mail: zhai@me.osakafu-u.ac.jp \\ ** Department of Electrical and Computer Engineering \\ Penn State Erie, Erie, PA 16563-1701, USA \\ e-mail: Xuping-Xu@psu .edu \\ *** Department of Electrical Engineering \\ University of Notre Dame \\ Notre Dame, Indiana 46556, USA \\ e-mail: hlin1@nd.edu \\ **** Department of Electrical and Computer Engineering \\ University of Illinois at Chicago \\ Chicago, IL 60607-7053, USA \\ e-mail: dliu@ece.uic.edu
}

\begin{abstract}
We analyze stability for switched systems which are composed of both continuous-time and discrete-time subsystems. By considering a Lie algebra generated by all subsystem matrices, we show that if all subsystems are Hurwitz/Schur stable and this Lie algebra is solvable, then there is a common quadratic Lyapunov function for all subsystems and thus the switched system is exponentially stable under arbitrary switching. When not all subsystems are stable and the same Lie algebra is solvable, we show that there is a common quadratic Lyapunov-like function for all subsystems and the switched system is exponentially stable under a dwell time scheme. Two numerical examples are provided to demonstrate the result.
\end{abstract}

Keywords: switched systems, common quadratic Lyapunov functions, Lie algebra, exponential stability, arbitrary switching, dwell time scheme.

\section{Introduction}

In the last two decades, there has been increasing interest in stability analysis and controller design for switched systems; see the survey papers (DeCarlo et al., 2000; Liberzon and Morse, 1999), the recent book (Liberzon, 2003) and the references cited therein. In (Liberzon and Morse, 1999; Liberzon, 2003), it is pointed out that there are three basic problems in stability and design of switched systems: (i) find conditions for stability under arbitrary switching, (ii) identify the limited but useful class of stabilizing switching laws, and (iii) construct a stabilizing switching law. The necessary condition for the first problem is that all subsystems are stable. When arbitrary switching cannot be guaranteed, including the case where unstable subsystems are involved, we have to consider the second and third problems.

In this paper, we focus our attention on the first problem. There are several existing works on Problem (i), most of which deal with the case where the switched systems are composed of continuous-time subsystems. Narendra and Balakrishnan (1994) showed that when all subsystems are stable and pairwise commutative, the switched linear system is stable under arbitrary switching. Liberzon et al. (1999) extended this result from the commutation condition to a Lie algebraic condition. Zhai (2003) showed that a class of switched symmetric systems are asymptotically 
stable under arbitrary switching since a common quadratic Lyapunov function, in the form of $V(x)=x^{T} x$, exists for all subsystems. The works (Zhai, 2003; Zhai et al., 2002b) extended the considerations to stability analysis problems for switched systems composed of discrete-time subsystems.

Motivated by the observation that all these papers deal with switched systems composed of only continuoustime subsystems or only discrete-time ones, Zhai et al. (2004) considered the new type of switched systems which are composed of both continuous-time and discrete-time dynamical subsystems. As was also pointed out in (Zhai et al., 2004), it is easy to find many applications involving this kind of switched systems, e.g., continuous-time systems with impulsive effects, where the impulsive effect/state jump can be modeled as discrete-time subsystems. Another example of a system of this kind is a continuous-time plant controlled either by a physically implemented regulator or by a digitally implemented one (and a switching rule between them). We also point out that a lot of other practical systems, including bipedal robots and networked control systems, can be modeled in this framework. Zhai et al. (2004) made some analysis and obtained design results for several kinds of such mixed-type switched systems. For example, in the case where a commutation condition holds, and the case of switched symmetric systems, it is shown that if all subsystems are stable, then there exists a common quadratic Lyapunov function for all subsystems and thus the switched system is exponentially stable under arbitrary switching. Recently, the authors extended the results for switched symmetric systems (Zhai et al., 2004) to switched normal systems (Zhai et al., 2006). For such switched systems, it is shown that when all continuous-time subsystems are Hurwitz stable and all discrete-time subsystems are Schur stable, a common quadratic Lyapunov function exists for the subsystems and that the switched system is exponentially stable under an arbitrary switching. Some discussions are also given for the case where unstable subsystems are involved.

In this paper, we aim to apply the Lie algebraic approach, proposed in (Liberzon et al., 1999; Agrachev and Liberzon, 2001), to switched systems which are composed of both continuous-time and discrete-time subsystems, where unstable subsystems may be included. By considering a Lie algebra generated by all subsystem matrices, we show that if all subsystems are Hurwitz/Schur stable and this Lie algebra is solvable, then there is a common quadratic Lyapunov function for all subsystems and thus the switched system is exponentially stable under arbitrary switching. In the case where not all subsystems are stable and the Lie algebra is solvable, we show that there is a common quadratic Lyapunov-like function for all subsystems and the switched system is exponentially stable with a reasonable decay rate under a dwell time scheme.
Two numerical examples are provided to demonstrate the results.

It is noted here that this paper is motivated mainly by three existing papers (Liberzon et al., 1999; Zhai et al., 2004; Zhai et al., 2001b). The extension of the Lie algebraic condition from the case where all subsystems are stable (Liberzon et al., 1999) to the case where unstable subsystems are included (Zhai et al., 2001b) is not trivial, and the same is true when dealing with the case where both continuous-time and discrete-time subsystems exist (Zhai et al., 2004). In fact, as will be seen later, we modify the proof in (Liberzon et al., 1999) to fit our present setting where both continuous-time and discretetime subsystems, and furthermore both stable and unstable subsystems, exist.

\section{System Description and Preliminaries}

We consider the switched system which is composed of a set of continuous-time LTI subsystems

$$
\dot{x}(t)=A_{c i} x(t), \quad i=1, \ldots, N_{c}
$$

and a set of discrete-time LTI subsystems

$$
x(k+1)=A_{d j} x(k), \quad j=1, \ldots, N_{d},
$$

where $x(t), x(k) \in \mathbb{R}^{n}$ are the subsystem states, and $A_{c i}$ 's and $A_{d j}$ 's are constant matrices of appropriate dimensions. $N_{c} \geq 1$ and $N_{d} \geq 1$ are the numbers of continuoustime subsystems and discrete-time ones, respectively.

Remark 1. Note that the above system is rather different from sampled-data systems which are usually composed of a continuous-time system and a discrete-time controller with some (time-invariant or time-variant) sampling periods. However, as an important application, the above setting can be adapted to sampled-data control. For example, for a physical system which may be subject to a long sampling period and a very short sampling period, we design a continuous-time controller for the very short sampling period and a discrete-time controller for the long sampling period. Then the two closed-loop systems can be regarded as a switched system composed of both continuous-time and discrete-time subsystems as (1) and (2).

To discuss the stability of the overall switched system, we assume for simplicity that the sampling periods of all the discrete-time subsystems are of the same value $\tau$ (the discussion can be easily extended to the case where the discrete-time subsystems have different sampling periods). Since the states of the discrete-time subsystems can be viewed as piecewise constant vectors between sampling points, we can consider the value of the entire system states in continuous-time domain. For example, if Subsystem $A_{c 1}$ is activated on $\left[t_{0}, t_{1}\right]$ and then Subsystem 
$A_{d 1}$ is activated for $m$ steps and Subsystem $A_{c 2}$ is activated from then to $t_{2}$, the time domain is divided into

$$
\left[t_{0}, t_{2}\right]=\left[t_{0}, t_{1}\right] \cup\left[t_{1}, t_{1}+m \tau\right] \cup\left[t_{1}+m \tau, t_{2}\right]
$$

and the system state takes the form of

$$
x(t)=\left\{\begin{array}{c}
e^{A_{c 1}\left(t-t_{0}\right)} x\left(t_{0}\right), t \in\left[t_{0}, t_{1}\right] \\
A_{d 1}^{k-1} x\left(t_{1}\right), \quad t \in\left[t_{1}+(k-1) \tau, t_{1}+k \tau\right), \\
1 \leq k \leq m \\
e^{A_{c 2}\left(t-t_{1}-m \tau\right)} A_{d 1}^{m} x\left(t_{1}\right), t \in\left[t_{1}+m \tau, t_{2}\right] .
\end{array}\right.
$$

Although $x(t)$ is not continuous with respect to time $t$ due to the existence of discrete-time subsystems, the solution $x(t)$ is uniquely defined at all time instants, and thus various stability properties can be discussed in the continuoustime domain. This kind of approach has been frequently used in the analysis and design of digital control systems.

Throughout this paper, we assume that at least one stable (continuous-time or discrete-time) subsystem exists among (1) and (2). Without loss of generality, we assume here that Subsystems $A_{c 1}, \ldots, A_{c s_{c}}\left(0 \leq s_{c} \leq N_{c}\right)$ and Subsystems $A_{d 1}, \ldots, A_{d s_{d}}\left(0 \leq s_{d} \leq N_{d}, s_{c}+s_{d} \geq 1\right)$ are stable while the others (if they exist) are not stable.

In the end of this section, we give some preliminaries of a Lie algebra. Most of the material is picked up from (Liberzon et al., 1999; Agrachev and Liberzon, 2001). Interested readers are referred to these or more detailed textbooks on Lie algebras (Gorbatsevich et al., 1994; Samelson, 1969).

A Lie algebra is a finite-dimensional vector space equipped with a Lie bracket, i.e., a bilinear, skewsymmetric map $\mathcal{L} \times \mathcal{L} \rightarrow \mathcal{L}$ satisfying the Jacobi identity $[a,[b, c]]+[b,[c, a]]+[c,[a, b]]=0$. In the case of a matrix Lie algebra, the standard Lie bracket is defined as

$$
[A, B] \triangleq A B-B A
$$

If $\mathcal{L}_{1}$ and $\mathcal{L}_{2}$ are linear subspaces of a Lie algebra $\mathcal{L}$, we write $\left[\mathcal{L}_{1}, \mathcal{L}_{2}\right]$ for the linear space spanned by all the products $\left[L_{1}, L_{2}\right]$ with $L_{1} \in \mathcal{L}_{1}$ and $L_{2} \in \mathcal{L}_{2}$, and we define the sequence $\mathcal{L}^{(k)}$ inductively as follows:

$$
\mathcal{L}^{(1)} \triangleq \mathcal{L}, \quad \mathcal{L}^{(k+1)} \triangleq\left[\mathcal{L}^{(k)}, \mathcal{L}^{(k)}\right] \subset \mathcal{L}^{(k)} .
$$

If $\mathcal{L}^{(k)}=0$ for some $k$ sufficiently large, then $\mathcal{L}$ is called solvable. For example, if $\mathcal{L}$ is a Lie algebra generated by two matrices $A$ and $B$, we have

$$
\begin{aligned}
\mathcal{L}^{(1)} & =\operatorname{span}\{A, B,[A, B],[A,[A, B]], \ldots\}, \\
\mathcal{L}^{(2)} & =\operatorname{span}\{[A, B],[A,[A, B]],[B,[A, B]], \ldots\}, \\
\mathcal{L}^{(3)} & =\operatorname{span}\{[[A, B],[A,[A, B]]], \ldots\},
\end{aligned}
$$

and so on.
The following result plays a key role in our subsequent discussion. It is known as Lie's theorem and can be found in most textbooks on Lie algebra theory (Gorbatsevich et al., 1994; Samelson, 1969).

Lemma 1. Let $\mathcal{L}$ be a solvable Lie algebra over an algebraically closed field, and let $\rho$ be a representation of $\mathcal{L}$ on a vector space $V$ of finite dimension $n$. Then, there exists a basis $\left\{v_{1}, v_{2}, \ldots, v_{n}\right\}$ of $V$ such that for each $X \in \mathcal{L}$ the matrix $\rho(X)$ in that basis takes the upper-triangular form

$$
\left[\begin{array}{ccc}
\lambda_{1}(X) & \cdots & * \\
\vdots & \ddots & \vdots \\
0 & \cdots & \lambda_{n}(X)
\end{array}\right]
$$

where $\lambda_{1}(X), \ldots, \lambda_{n}(X)$ are its eigenvalues.

This lemma will be used in Section 4 for the Lie algebra composed of all subsystem matrices in the switched system.

\section{Stability Analysis Using CQLF/CQLLF}

In this section, we discuss the stability of the switched system using the approach of common quadratic Lyapunov (or Lyapunov-like) functions.

Definition 1. If all the subsystems are Hurwitz/Schur stable (i.e., $s_{c}=N_{c}, s_{d}=N_{d}$ ) and there is a common positive definite matrix $P$ satisfying

$$
\begin{array}{ll}
A_{c i}^{T} P+P A_{c i}<0, & i=1, \ldots, N_{c}, \\
A_{d j}^{T} P A_{d j}-P<0, & j=1, \ldots, N_{d},
\end{array}
$$

then $V(x)=x^{T} P x$ is called a common quadratic Lyapunov function (CQLF) for all the subsystems.

It is easy to see that when a CQLF exists, there are two positive scalars $\alpha_{c s}$ and $\alpha_{d s}<1$ such that

$$
\begin{aligned}
A_{c i}^{T} P+P A_{c i} & <-2 \alpha_{c s} P, \\
A_{d j}^{T} P A_{d j}-\alpha_{d s}^{2} P & <0
\end{aligned}
$$

hold for all $i$ and $j$.

Remark 2. Obviously, the necessary condition for the existence of a CQLF is that all continuous-time subsystems are Hurwitz stable and all discrete-time subsystems are Schur stable. There are many switched systems in which all the subsystems have a CQLF. For example, we showed (Zhai, 2003) that if all the subsystems are (Hurwitz or Schur) stable and symmetric, then $V(x)=x^{T} x$ $(P=I)$ is a CQLF. The result was extended in (Zhai $e t$ $a l ., 2006)$ to switched normal systems $\left(A_{c i}^{T} A_{c i}=A_{c i} A_{c i}^{T}\right.$, $A_{d j}^{T} A_{d j}=A_{d j} A_{d j}^{T}$ ). In (Zhai et al., 2004), we proved constructively that if all the subsystems are (Hurwitz or 
Schur) stable and commutative pariwise, then there exists a CQLF for all the subsystems.

Theorem 1. If there is a CQLF for all the subsystems, the switched system composed of (1) and (2) is exponentially stable under arbitrary switching.

Proof. To show exponential stability, we first find positive scalars $\alpha_{c s}$ and $\alpha_{d s}$ satisfying (10). Then, in the period where a continuous-time subsystem is activated, we obtain $\dot{V}(x(t))<-2 \alpha_{c s} V(x(t))$, and in the period where a discrete-time subsystem is activated, $V(x(k+1))<$ $\alpha_{d s}^{2} V(x(k))$.

For any time $t>0$ (when a discrete-time subsystem is active at $t$, we refer to $t$ tacitly as the last sampling point since the state does not change until the next sampling point), we can always divide the time interval $[0, t]$ as $t=t_{c}+m \tau(m \geq 0)$, where $t_{c}$ is the total duration time on continuous-time subsystems and $m \tau$ is the total duration time on discrete-time subsystems. It is not difficult to obtain that whatever the activation order is,

$$
V(x(t)) \leq e^{-2 \alpha_{c s} t_{c}} \alpha_{d s}^{2 m} V(x(0))
$$

and thus

$$
|x(t)| \leq \sqrt{\frac{\lambda_{M}(P)}{\lambda_{m}(P)}} e^{-\alpha_{s} t}|x(0)|,
$$

where

$$
\alpha_{s}=\min \left\{\alpha_{c s}, \frac{\ln \left(\alpha_{d s}^{-1}\right)}{\tau}\right\}>0,
$$

$\lambda_{M}(P)$ and $\lambda_{m}(P)$ denote the largest and smallest eigenvalues of $P$, respectively. Since we did not add any limitation on the switching signals, the switched system is exponentially stable under arbitrary switching.

Now, we deal with the case where not all the subsystems are Hurwitz/Schur stable. To proceed, we need the following definition:

Definition 2. If not all the subsystems are Hurwitz/Schur stable and there is a common positive definite matrix $P$ satisfying

$$
\begin{gathered}
\left(A_{c i}+\alpha_{c s} I\right)^{T} P+P\left(A_{c i}+\alpha_{c s} I\right)<0, \\
i=1, \ldots, N_{c s_{c}}, \\
\left(A_{c i}-\alpha_{c u} I\right)^{T} P+P\left(A_{c i}-\alpha_{c u} I\right)<0, \\
i>N_{c s_{c}}, \\
\left(\frac{A_{d j}}{\alpha_{d s}}\right)^{T} P\left(\frac{A_{d j}}{\alpha_{d s}}\right)-P<0, \quad j=1, \ldots, N_{d s_{d}}, \\
\left(\frac{A_{d j}}{\alpha_{d u}}\right)^{T} P\left(\frac{A_{d j}}{\alpha_{d u}}\right)-P<0, \quad j>N_{d s_{d}},
\end{gathered}
$$

with scalars $\alpha_{c s}>0, \alpha_{c u}>0,0<\alpha_{d s}<1$ and $\alpha_{d u}>$ 1 , then $V(x)=x^{T} P x$ is called a common quadratic Lyapunov-like function (CQLLF) for all the subsystems.

Remark 3. In Definition 2, the matrix inequalities (13) and (15) are the same as (10), which corresponds to the stable subsystems, while (14) and (16) are for the unstable subsystems. For any unstable $A_{c i}$, it is easy to find positive real $\alpha_{c u}$ such that $A_{c i}-\alpha_{c u} I$ is stable. The same holds with unstable $A_{d j}$. The key point is that all the obtained stable matrices must correspond to a common positive definite matrix.

Now, let us investigate what happens when Definition 2 is true. According to the matrix inequalities in Definition 2 , in the period where a continuous-time subsystem is activated we obtain

$$
\dot{V}(x(t))< \begin{cases}-2 \alpha_{c s} V(x(t)) & \text { when the subsystem } \\ & \text { is stable, } \\ 2 \alpha_{c u} V(x(t)) & \text { when the subsystem } \\ & \text { is unstable. }\end{cases}
$$

Similarly, in the period where a discrete-time subsystem is activated we obtain

$$
\begin{aligned}
& V(x(k+1)) \begin{cases}\alpha_{d s}^{2} V(x(k)) & \text { when the subsystem } \\
\text { is stable, }\end{cases} \\
& \alpha_{d u}^{2} V(x(k)) \text { when the subsystem } \\
& \text { is unstable. }
\end{aligned}
$$

For any time $t>0$, we can always divide the time interval $[0, t]$ as $t=t_{c s}+t_{c u}+\left(m_{s}+m_{u}\right) \tau$, where $t_{c s}$ and $t_{c u}$ are the total duration times on stable and unstable continuous-time subsystems, respectively, and $m_{s} \tau$ and $m_{u} \tau$ are the total duration times on stable and unstable discrete-time subsystems, respectively. Then it is not difficult to obtain that whatever the activation order is,

$$
V(x(t)) \leq e^{-2 \alpha_{c s} t_{c s}+2 \alpha_{c u} t_{c u}} \alpha_{d s}^{2 m_{s}} \alpha_{d u}^{2 m_{u}} V(x(0)) .
$$

Defining

$$
\begin{aligned}
& \alpha_{s}=\min \left\{\alpha_{c s}, \frac{\ln \left(\alpha_{d s}^{-1}\right)}{\tau}\right\}>0, \\
& \alpha_{u}=\max \left\{\alpha_{c u}, \frac{\ln \left(\alpha_{d u}\right)}{\tau}\right\}>0,
\end{aligned}
$$

we obtain

$$
V(x(t)) \leq e^{-2 \alpha_{s} t_{s}+2 \alpha_{u} t_{u}} V(x(0)),
$$

where $t_{s}=t_{c s}+m_{s} \tau$ is the total duration time on stable subsystems and $t_{u}=t_{c u}+m_{u} \tau$ is the total duration time on unstable subsystems. 
For any given positive scalar $\alpha<\alpha_{s}$, we consider the following dwell time scheme:

$$
\frac{t_{u}}{t_{s}} \leq \frac{\alpha_{s}-\alpha}{\alpha_{u}+\alpha}
$$

which specifies an upper bound to the distance between the total duration time on unstable subsystems and stable ones.

Since (21) is equivalent to

$$
-2 \alpha_{s} t_{s}+2 \alpha_{u} t_{u} \leq-2 \alpha\left(t_{s}+t_{u}\right)=-2 \alpha t,
$$

from $(20)$ we obtain

$$
|x(t)| \leq \sqrt{\frac{\lambda_{M}(P)}{\lambda_{m}(P)}} e^{-\alpha t}|x(0)| .
$$

We summarize the above discussion in the following theorem:

Theorem 2. If there is a CQLLF for all the subsystems satisfying (13)-(16), the switched system composed of (17) and (2) is exponentially stable with decay rate $\alpha$ under the dwell time scheme (21).

Remark 4. If we desire the decay rate $\alpha_{s}$, then according to 21) the total activation time on unstable subsystems $\left(t_{u}\right)$ must be zero, which means that unstable subsystems are not activated. This is in accordance with Theorem 1. Further, $\alpha$ can be very close to $\alpha_{s}$ if $t_{u}$ is chosen sufficiently small, which is also reasonable.

\section{Lie Algebraic Conditions}

We first state a result which will be used later in the proof of the main theorem.

Lemma 2. All leading principal minors of a Hermitian matrix are real. A Hermitian matrix $H$ is positive definite (i.e., $x^{*} H x>0, \forall x \neq 0$ ) if and only if all its leading principal minors are positive.

Now, we state and prove the first main theorem of this section.

Theorem 3. If all the subsystems are Hurwitz/Schur stable and the Lie algebra

$$
\left\{A_{c i}, i=1, \ldots, N_{c} ; A_{d j}, j=1, \ldots, N_{d}\right\}_{L A}
$$

is solvable, then there exists a CQLF for all the subsystems, and thus the switched system composed of (1) and (2) is exponentially stable under arbitrary switching.

Proof. According to Theorem 1, the proof is reduced to finding a CQLF for all the subsystems. Without loss of generality, we assume here for simplicity that $n=3$.
Translating Lemma 1 into the present situation, we see that if the Lie algebra (24) is solvable, then there exists a nonsingular complex matrix $U$ such that for all $i$ and $j$ we have

$$
A_{c i}=U^{-1} \tilde{A}_{c i} U, \quad A_{d j}=U^{-1} \tilde{A}_{d j} U,
$$

where the complex matrices $\tilde{A}_{c i}$ and $\tilde{A}_{d j}$ are uppertriangular.

We first show that there exists a real positive definite matrix $\tilde{P}$ such that

$$
\tilde{A}_{c i}^{*} \tilde{P}+\tilde{P} \tilde{A}_{c i}<0, \quad \tilde{A}_{d j}^{*} \tilde{P} \tilde{A}_{d j}-\tilde{P}<0 .
$$

Especially, we choose $\tilde{P}$ with a real diagonal form as $\tilde{P}=$ $\operatorname{diag}\left\{\tilde{p}_{1}, \tilde{p}_{2}, \tilde{p}_{3}\right\}$, and thus we have

$$
\begin{aligned}
& -\tilde{A}_{c i}^{*} \tilde{P}-\tilde{P} \tilde{A}_{c i} \\
& =\left[\begin{array}{ccc}
-2 \tilde{p}_{1}\left(\Re \tilde{A}_{c i}\right)_{11} & -\tilde{p}_{1}\left(\tilde{A}_{c i}\right)_{12} & -\tilde{p}_{1}\left(\tilde{A}_{c i}\right)_{13} \\
* & -2 \tilde{p}_{2}\left(\Re \tilde{A}_{c i}\right)_{22} & -\tilde{p}_{2}\left(\tilde{A}_{c i}\right)_{23} \\
* & * & -2 \tilde{p}_{3}\left(\Re \tilde{A}_{c i}\right)_{33}
\end{array}\right]
\end{aligned}
$$

and

$$
\begin{aligned}
& -\tilde{A}_{d j}^{*} \tilde{P} \tilde{A}_{d j}+\tilde{P} \\
& =\left[\begin{array}{cc}
\tilde{p}_{1}\left(1-\left|\left(\tilde{A}_{d j}\right)_{11}\right|^{2}\right) & -\tilde{p}_{1}\left(\tilde{A}_{d j}\right)_{11}^{*}\left(\tilde{A}_{d j}\right)_{12} \\
* & \tilde{p}_{2}\left(1-\left|\left(\tilde{A}_{d j}\right)_{22}\right|^{2}\right)-\tilde{p}_{1}\left|\left(\tilde{A}_{d j}\right)_{12}\right|^{2} \\
* & *
\end{array}\right. \\
& -\tilde{p}_{1}\left(\tilde{A}_{d j}\right)_{11}^{*}\left(\tilde{A}_{d j}\right)_{13} \\
& -\tilde{p}_{1}\left(\tilde{A}_{d j}\right)_{12}^{*}\left(\tilde{A}_{d j}\right)_{13}-\tilde{p}_{2}\left(\tilde{A}_{d j}\right)_{22}^{*}\left(\tilde{A}_{d j}\right)_{23} \\
& \left.\tilde{p}_{3}\left(1-\left|\left(\tilde{A}_{d j}\right)_{33}\right|^{2}\right)-\tilde{p}_{1}\left|\left(\tilde{A}_{d j}\right)_{13}\right|^{2}-1 \tilde{p}_{2}\left|\left(\tilde{A}_{d j}\right)_{23}\right|^{2}\right]
\end{aligned}
$$

Since all the subsystems are assumed to be Hurwitz/Schur stable, $\left(\Re \tilde{A}_{c i}\right)_{11}<0$ and $\left|\left(\tilde{A}_{d j}\right)_{11}\right|<1$ hold for all $i$ and $j$. Then we can choose positive $\tilde{p}_{1}$ arbitrarily so that the first leading principal minors of (27) and (28), namely, $-2 \tilde{p}_{1}\left(\Re \tilde{A}_{c i}\right)_{11}$ and $\tilde{p}_{1}\left(1-\left|\left(\tilde{A}_{d j}\right)_{11}\right|^{2}\right)$ are both positive.

Next, since $\left(\Re \tilde{A}_{c i}\right)_{22}<0$ and $\left|\left(\tilde{A}_{d j}\right)_{22}\right|<1$, we can always find a sufficiently large positive scalar $\tilde{p}_{2}$ such that for fixed $\tilde{p}_{1}$, the second leading principal minors of 27) and (28), namely, both

$$
\left|\begin{array}{cc}
-2 \tilde{p}_{1}\left(\Re \tilde{A}_{c i}\right)_{11} & -\tilde{p}_{1}\left(\tilde{A}_{c i}\right)_{12} \\
* & -2 \tilde{p}_{2}\left(\Re \tilde{A}_{c i}\right)_{22}
\end{array}\right|
$$

and

$$
\left|\begin{array}{cc}
\tilde{p}_{1}\left(1-\left|\left(\tilde{A}_{d j}\right)_{11}\right|^{2}\right) & -\tilde{p}_{1}\left(\tilde{A}_{d j}\right)_{11}^{*}\left(\tilde{A}_{d j}\right)_{12} \\
* & \tilde{p}_{2}\left(1-\left|\left(\tilde{A}_{d j}\right)_{22}\right|^{2}\right)-\tilde{p}_{1}\left|\left(\tilde{A}_{d j}\right)_{12}\right|^{2}
\end{array}\right|
$$


are positive.

Finally, since $\left(\Re \tilde{A}_{c i}\right)_{33}<0$ and $\left|\left(\tilde{A}_{d j}\right)_{33}\right|<1$, for fixed $\tilde{p}_{1}$ and $\tilde{p}_{2}$, we can always find a sufficiently large positive scalar $\tilde{p}_{3}$ such that the third leading principal minors (i.e., the determinants) of (27) and (28) are both positive.

In this way, we have chosen $\tilde{p}_{1}, \tilde{p}_{2}$ and $\tilde{p}_{3}$ so that all the leading principal minors of (27) and 28) are positive. Therefore, according to Lemma 2, 26 is satisfied with the chosen $\tilde{P}$. Using the obtained $\tilde{P}$, we substitute 25) into (26) to obtain

$$
\begin{aligned}
& \tilde{P} U A_{c i} U^{-1}+\left(U^{-1}\right)^{*} A_{c i}^{T} U^{*} \tilde{P}<0 \\
& \left(U^{-1}\right)^{*} A_{d j}^{T} U^{*} \tilde{P} U A_{d j} U^{-1}-\tilde{P}<0
\end{aligned}
$$

which are respectively equivalent to

$$
P A_{c i}+A_{c i}^{T} P<0, \quad A_{d j}^{T} P A_{d j}-P<0,
$$

where $P=U^{*} \tilde{P} U$.

We write the complex matrix $P$ as $P=\Re(P)+$ $\sqrt{-1} \Im(P)$. Since $P$ is Hermitian, $\Im(P)$ is skewsymmetric, from which $x^{T} P x=x^{T} \Re(P) x>0(x \neq 0)$ is obtained. Thus, $\Re(P)$ is a real positive definite matrix. Similarly, we can easily obtain

$$
\begin{aligned}
& A_{c i}^{T} \Re(P)+\Re(P) A_{c i}<0, \\
& A_{d j}^{T} \Re(P) A_{d j}-\Re(P)<0,
\end{aligned}
$$

which implies that $\Re(P)$ is the common Lyapunov matrix we want to compute. This completes the proof.

Remark 5. It is understood from the proof of Theorem 3 that the result can be extended to the case where both upper-triangular and lower-triangular $\tilde{A}_{c i}$ 's (or $\tilde{A}_{d j}$ 's) exist. This means that if the Lie algebra (24) is not solvable, we can try to replace some subsystem matrices with their transposes in (24) and then check the new Lie algebra.

Remark 6. As was also pointed out in (Liberzon et al., 1999), although we have showed the existence condition of CQLF constructively, the computation depends on the value of the transformation matrix $U$. Since obtaining the value of $U$ may need some efforts when using standard numerical methods, it may be more efficient to solve the LMIs (8) and (9) with respect to $P>0$ directly, using the existing LMI software or the stochastic method proposed in (Liberzon and Tempo, 2003).

Next, we deal with the case where not all the subsystems are stable.

Theorem 4. If not all the subsystems are stable and the Lie algebra (24) is solvable, then there exists a CQLLF for all the subsystems and thus the switched system composed of (1) and (2) is exponentially stable with decay rate $\alpha$ under the dwell time scheme (21).
Proof. Since the Lie algebra (24) is solvable, there exists a nonsigular complex matrix $U$ such that (25) holds for all $i, j$, where $\tilde{A}_{c i}, \tilde{A}_{d j}$ are upper-triangular.

For Hurwitz stable $A_{c i}$ s, there always exists a positive scalar $\alpha_{c s}$ such that $A_{c i}+\alpha_{c s} I$ remains Hurwitz stable. For Hurwitz unstable $A_{c i}$ 's, there always exists a positive scalar $\alpha_{c u}$ such that $A_{c i}-\alpha_{c u} I$ is Hurwitz stable. Similarly, for discrete-time subsystems, we find a positive scale $\alpha_{d s}<1$ for Schur stable $A_{d j}$ such that $A_{d j} / \alpha_{d s}$ remains Schur stable, and $\alpha_{d u}>1$ for unstable $A_{d j}$ such that $A_{d j} / \alpha_{d u}$ becomes Schur stable.

From 25 we compute

$$
\begin{aligned}
& A_{c i}+\alpha_{c s} I=U^{-1}\left(\tilde{A}_{c i}+\alpha_{c s} I\right) U, i=1, \ldots, N_{c s_{c}} \\
& A_{c i}-\alpha_{c u} I=U^{-1}\left(\tilde{A}_{c i}-\alpha_{c u} I\right) U, \quad i>N_{c s_{c}} \\
& \frac{A_{d j}}{\alpha_{d s}}=U^{-1} \frac{\tilde{A}_{d j}}{\alpha_{d s}} U, \quad j=1, \ldots, N_{d s_{d}} \\
& \frac{A_{d j}}{\alpha_{d u}}=U^{-1} \frac{\tilde{A}_{d j}}{\alpha_{d u}} U, \quad j>N_{d s_{d}}
\end{aligned}
$$

and note that all the matrices $\tilde{A}_{c i}+\alpha_{c s} I\left(1 \leq i \leq N_{c s_{c}}\right)$, $\tilde{A}_{c i}-\alpha_{c u} I\left(N_{c s_{c}}<i \leq N_{c}\right), \tilde{A}_{d j} / \alpha_{d s}\left(1 \leq j \leq N_{d s_{d}}\right)$ and $\tilde{A}_{d j} / \alpha_{d u}\left(N_{d s_{d}}<j \leq N_{d}\right)$ are Hurwitz/Schur stable and are still upper-triangular.

Then, using the same technique as in the proof of Theorem 3, we can obtain a common positive definite (diagonal) matrix $P$ for $A_{c i}+\alpha_{c s} I\left(1 \leq i \leq N_{c s_{c}}\right)$, $A_{c i}-\alpha_{c u} I\left(N_{c s_{c}}<i \leq N_{c}\right), A_{d j} / \alpha_{d s}\left(1 \leq j \leq N_{d s_{d}}\right)$ and $A_{d j} / \alpha_{d u}\left(N_{d s_{d}}<j \leq N_{d}\right)$. This completes the proof of the existence of a CQLLF. According to Theorem 2, the switched system is exponentially stable with decay rate $\alpha$ under the dwell time scheme 21.

\section{Examples}

Example 1. Consider the switched system with one continuous-time subsystem and one discrete-time subsystem whose system matrices are

$$
A_{c}=\left[\begin{array}{rr}
-0.5 & -0.5 \\
0.1 & -0.3
\end{array}\right], \quad A_{d}=\left[\begin{array}{ll}
0.4 & 0.2 \\
0.2 & 0.3
\end{array}\right]
$$

It is easy to confirm that $A_{c}$ is Hurwitz stable and $A_{d}$ is Schur stable.

Some standard Lie brackets are computed as

$$
\begin{aligned}
{\left[A_{c}, A_{d}\right] } & =\left[\begin{array}{rr}
-0.1200 & 0.0100 \\
0.0500 & 0.1200
\end{array}\right], \\
{\left[A_{c},\left[A_{c}, A_{d}\right]\right] } & =\left[\begin{array}{rr}
-0.0260 & -0.1220 \\
-0.0140 & 0.0260
\end{array}\right],
\end{aligned}
$$




$$
\begin{aligned}
{\left[A_{d},\left[A_{c}, A_{d}\right]\right] } & =\left[\begin{array}{rr}
-0.0080 & 0.0490 \\
-0.0530 & -0.0080
\end{array}\right], \\
{\left[\left[A_{c}, A_{d}\right],\left[A_{c},\left[A_{c}, A_{d}\right]\right]\right] } & =\left[\begin{array}{rr}
-0.0060 & -0.0298 \\
-0.0060 & 0.0060
\end{array}\right], \\
{\left[\left[A_{c}, A_{d}\right],\left[A_{d},\left[A_{c}, A_{d}\right]\right]\right] } & =\left[\begin{array}{rr}
-0.0030 & -0.0119 \\
-0.0119 & 0.0030
\end{array}\right],
\end{aligned}
$$

and

$$
\begin{gathered}
{\left[\left[\left[A_{c}, A_{d}\right],\left[A_{c},\left[A_{c}, A_{d}\right]\right]\right],\left[\left[A_{c}, A_{d}\right],\left[A_{d},\left[A_{c}, A_{d}\right]\right]\right]\right]} \\
=\left[\begin{array}{rr}
-0.0004 & 0.0000 \\
0.0001 & 0.0004
\end{array}\right] .
\end{gathered}
$$

It is seen from further computations that the Lie algebra $\left\{A_{c}, A_{d}\right\}_{L A}$ is solvable with $k=5$, and thus the switched system is exponentially stable under arbitrary switching.

Suppose that the sampling period of Subsystem $A_{d}$ is 0.01 . Figure 1 shows the convergence of the system state and the norm when $A_{c}$ and $A_{d}$ are activated alternatively with a randomly generated time series (0.5, 3 steps, $0.6,2$ steps, $0.25,4$ steps) with the initial state $\left[\begin{array}{ll}100 & 100\end{array}\right]^{T}$. The asterisk in the left part of Fig. 1 describes the discretetime state change, and the right part of Fig. 1 shows that the norm of the system state converges to zero quickly.

Example 2. Modify the matrix $A_{c}$ in Example 1 and use the same $A_{d}$ as

$$
A_{c}=\left[\begin{array}{rr}
0 & -0.5 \\
0.1 & 0.2
\end{array}\right], A_{d}=\left[\begin{array}{ll}
0.4 & 0.2 \\
0.2 & 0.3
\end{array}\right] .
$$

It is easy to confirm that $A_{c}$ in the above equation is equal to the matrix $A_{c}$ in (38) plus $0.5 I$, and thus the Lie alge-
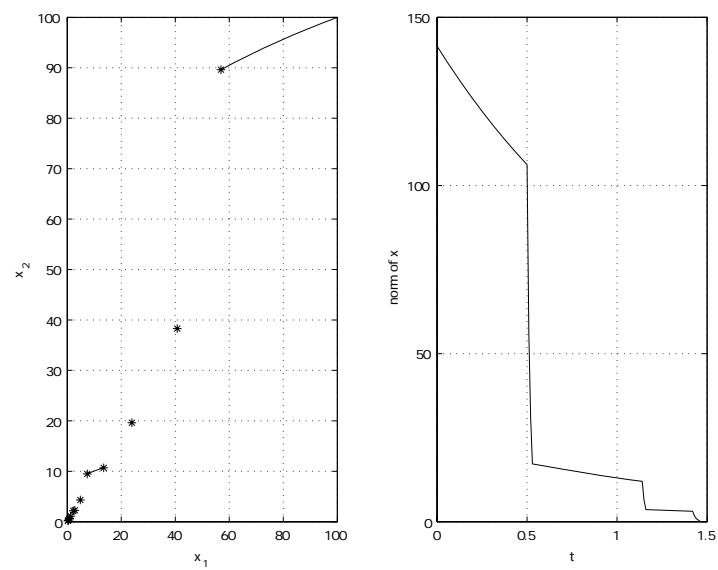

Fig. 1. System state and its norm in Example 1. bra $\left\{A_{c}, A_{d}\right\}_{L A}$ here is also solvable without any need to compute the Lie brackets.

Since $A_{c}$ 's eigenvalues are $0.1 \pm 0.2 \sqrt{-1}$, we set $\alpha_{c u}=0.2$ to make $A_{c}-\alpha_{c u} I$ Hurwitz stable. Also, since $A_{d}$ 's eigenvalues are $(0.7 \pm \sqrt{0.17}) / 2$, we set $\alpha_{d s}=0.9$ so that $A_{d} / \alpha_{d s}$ remains Schur stable. Defining the sampling period as $\tau=0.1$, we obtain $\alpha_{s}=\ln \left(\alpha_{d s}^{-1}\right) / \tau=$ 1.054 and $\alpha_{u}=\alpha_{c u}=0.2$. If we choose the decay rate as $\alpha=0.1$, then according to 21], the dwell time scheme is $t_{u} / t_{s} \leq\left(\alpha_{s}-\alpha\right) /\left(\alpha_{u}+\alpha\right) \approx 3.17$. To satisfy this requirement, we choose activating $A_{c}$ with 3.1 and then $A_{d}$ with 10 steps, alternately. Figure 2 shows the convergence of the system state and the norm, where the initial state is $\left[\begin{array}{ll}200 & 100\end{array}\right]^{T}$.
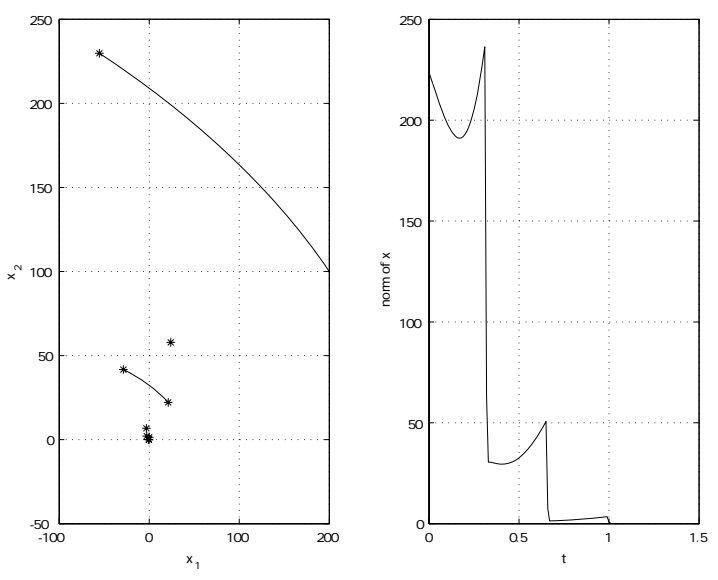

Fig. 2. System state and its norm in Example 2.

\section{Conclusion}

Lie algebraic conditions have been established for the stability of a class of switched systems, where both continuous-time and discrete-time linear subsystems exist and unstable subsystems may be included. The results are theoretically attractive, and the computation is not involved. Future work includes extention to the case of nonlinear subsystems.

\section{Acknowledgment}

The authors would like to thank Tony Michel from the University of Notre Dame and Joe Imae and Tomoaki Kobayashi from Osaka Prefecture University for their valuable discussions.

\section{References}

Agrachev A.A. and Liberzon D. (2001): Lie-algebraic stability criteria for switched systems. SIAM Journal on Control and Optimization, Vol. 40, No. 1, pp. 253-269. 
DeCarlo R., Branicky M.S., Pettersson S. and Lennartson B. (2000): Perspectives and results on the stability and stabilizability of hybrid systems. Proceedings of the IEEE, Vol. 88, No. 7, pp. 1069-1082.

Gorbatsevich V.V., Onishchik A.L. and Vinberg E.B. (1994): Structure of Lie Groups and Lie Algebras. Berlin: Springer.

Liberzon D. and Tempo R. (2003): Gradient algorithm for finding common Lyapunov functions. Proceedings of the 42nd IEEE Conference on Decision and Control, Hawaii, USA, pp. 4782-4787.

Liberzon D. (2003): Switching in Systems and Control. Boston: Birkhäuser.

Liberzon D. and Morse A.S. (1999): Basic problems in stability and design of switched systems. IEEE Control Systems Magazine, Vol. 19, No. 5, pp. 59-70.

Liberzon D., Hespanha J.P. and Morse A.S. (1999): Stability of switched systems: A Lie-algebraic condition. Systems and Control Letters, Vol. 37, No. 3, pp. 117-122.

Narendra K.S. and Balakrishnan J. A common Lyapunov function for stable LTI systems with commuting A-matrices. IEEE Transactions on Automatic Control, Vol. 39, No. 12, pp. 2469-2471.

Samelson H. (1969): Notes on Lie Algebra. New York: Van Nostrand Reinhold.

Zhai G. (2003): Stability and $\mathcal{L}_{2}$ gain analysis of switched symmetric systems, In: Stability and Control of Dynamical Systems with Applications, (D. Liu and P.J. Antsaklis, Eds.), Boston: Birkhäuser, pp. 131-152.
Zhai G. (2001a): Quadratic stabilizability of discrete-time switched systems via state and output feedback. Proceedings of the 40th IEEE Conference on Decision and Control, Orlando, FL, pp. 2165-2166.

Zhai G., Hu B., Yasuda K. and Michel A.N. (2002a): Stability and $\mathcal{L}_{2}$ gain analysis of discrete-time switched systems. Transactions of the Institute of Systems, Control and Information Engineers, Vol. 15, No. 3, pp. 117-125.

Zhai G., Chen X., Ikeda M. and Yasuda K. (2002b): Stability and $\mathcal{L}_{2}$ gain analysis for a class of switched symmetric systems. Proceedings of the 41st IEEE Conference on Decision and Control, Las Vegas, NV, pp. 4395-4400.

Zhai G., Lin H., Michel A.N., and Yasuda K. (2004): Stability analysis for switched systems with continuous-time and discrete-time subsystems. Proceedings of the American Control Conference, Boston, MA, pp. 4555-4560.

Zhai G., Xu X., Lin H., and Michel A.N. (2006): Analysis and design of switched normal systems. Nonlinear Analysis, Vol. 65, No. 12, pp. 2248-2259.

Zhai G., Hu B., Yasuda K. and Michel A.N. (2001b): Stability analysis of switched systems with stable and unstable subsystems: An average dwell time approach. International Journal of Systems Science, Vol. 32, No. 8, pp. 1055-1061.

Received: 10 April 2007

Revised: 20 October 2007 